\title{
Multifocal initiation and local propagation model of ALS
}

Clinical symptoms in most patients with amyotrophic lateral sclerosis (ALS) are focal at onset and spread contiguously. Some researchers, therefore, suggest that ALS pathology is similarly initiated at a single site and spreads via cell-to-cell propagation of prion-like pathogenic proteins, which act as misfolding templates. However, this 'single seed and simple propagation' model of ALS has been challenged by the findings of a new study indicating that abnormal lower motor neuron activity shows a noncontiguous pattern in many patients.

Teruhiko Sekiguchi and colleagues analysed the abnormal spontaneous activity in lower motor neurons of 36 patients with early-stage sporadic ALS using needle electromyography (EMG). To control for potential differences in motor neuronal vulnerability to ALS pathology, the team recorded needle EMG activity from pairs of muscles innervated from different spinal segments, but with similar innervating axon lengths and similar proportions of type I and type II muscle fibres. According to the 'single seed' hypothesis, such muscles should have similar

\section{The distribution ... cannot be explained by the 'single seed and simple propagation' hypothesis alone... 77}

intrinsic motor neuronal vulnerability to ALS pathology.

Only three patients showed contiguous patterns of needle EMG abnormalities. Another 19 patients had diffuse patterns, suggestive of relatively advanced disease. The remainder (14 patients) showed a noncontiguous distribution of anomalous needle EMG activity. Although Sekiguchi et al. did observe local clustering of needle EMG abnormalities, in accordance with the 'simple propagation' hypothesis, they assert that this skipping pattern of rostrocaudal spread does not support the 'single seed' hypothesis. "The distribution of lower [motor neuron] involvement cannot be explained by the 'single seed and simple propagation' hypothesis alone," their report notes. "We propose a 'multifocal hits and local propagation' hypothesis instead."

The investigators acknowledge that false-negative needle EMG readings in seemingly normal intermediate segments might explain the observed noncontiguous patterns. One potential source of falsenegative findings is that even if the pathological molecular processes occur simultaneously, the vulnerability of lower motor neurons to ALS pathology might differ, meaning that some motor neurons will degenerate more slowly than others and appear normal despite ongoing pathological changes. The researchers argue, however, that their selection of muscles that should have similar motor neuron vulnerability to ALS according to the prion-like model of propagation should minimize this limitation.

Sekiguchi et al. note that previous studies have reported that up to $30 \%$ of patients with sporadic ALS show noncontiguous spread of symptoms. The authors conclude that the observed noncontiguous pattern can only be explained by multifocal onset.

\section{Hemi Malkki}

Original article Sekiguchi, T. et al. Spreading of amyotrophic lateral sclerosis lesions-multifocal hits and local propagation? J. Neurol. Neurosurg. Psychiatry doi:10.1136/jnnp-2013-305617 